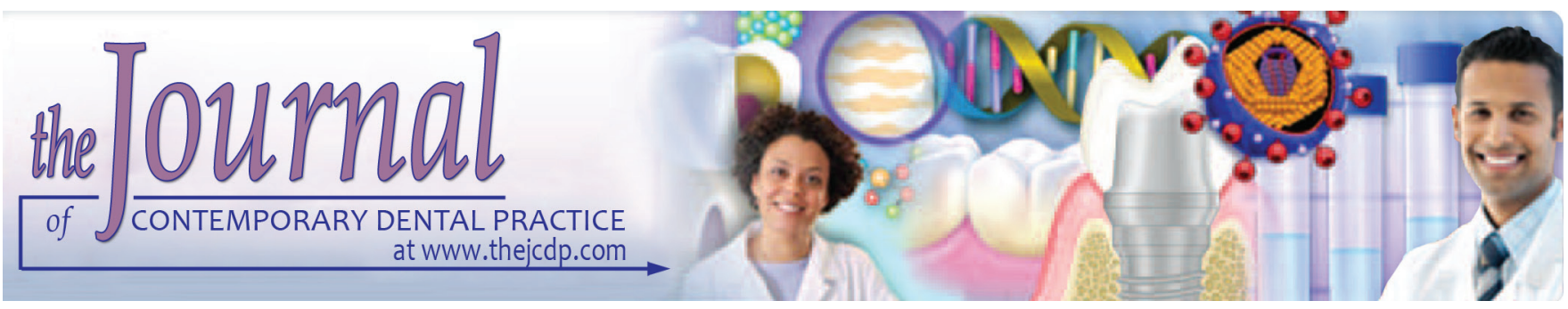

\title{
Mini-implants for Orthodontic Anchorage: Surface Analysis after Redrilling and Sterilization - An in vitro Study
}

\author{
1JM Gross, ${ }^{2}$ GG Nascimento, ${ }^{3}$ VC Araújo, ${ }^{4} \mathrm{MJS}$ Bönecker, ${ }^{5} \mathrm{C}$ Furuse
}

\begin{abstract}
Objective: This study aimed to investigate, in vitro, possible alterations on mini-implants surface after retrieval and if the cleaning process and sterilization can predispose damages.
\end{abstract}

Materials and methods: Two commercial mini-implants were tested for deformations after drilling and removing in artificial bone four times. Samples were analyzed by scanning electron microscopy, and surface alterations verified through thread and pitches deformation. To alterations caused by insertion/removal and the cleaning process and sterilization were verified in different procedures: Insertions and sterilization, only insertions, and only sterilization. Photomicrographs were analyzed in order to compare the surface characteristics. Head deformation was verified qualitatively. For a quantitative analysis, distances between threads were measured across the active part of the mini-implants.

Results: No deformation was observed in both groups. The cleaning and sterilization processes did not provoke alteration in both groups. Nevertheless, the presence of synthetic bone was noted in some samples. The mean distances between implant threads were similar after all steps in all regions in both groups.

Conclusion: The results suggest that the tested mini-implants can be retrieved without damage of its surface after four cycles of insertion, removal, and sterilization.

Keywords: Orthodontic mini-implant, Redrilling, Sterilization.

\footnotetext{
${ }^{1}$ Department of Orthodontics, Faculdade IPPEO, Curitiba Parana, Brazil

${ }^{2}$ Postgraduate Program in Dentistry, Federal University of Pelotas, Pelotas, Rio Grande do Sul, Brazil

${ }^{3}$ Instituto e Centro de Pesquisas São Leopoldo Mandic Campinas, São Paulo, Brazil

${ }^{4}$ School of Dentistry, University of São Paulo, São Paulo, Brazil

${ }^{5}$ School of Dentistry, São Paulo State University, Araçatuba São Paulo, Brazil

Corresponding Author: C Furuse, Assistant Professor School of Dentistry, São Paulo State University, CEP 16015-050 Rua José Bonifácio Araçatuba, São Paulo, Brazil, Phone: +551836363200, e-mail: cfuruse@foa.unesp.br
}

Clinical significance: Mini-implants can be retrieved without damage to its surface after four cycles of insertion, removal, and sterilization in the same patient without representing a biological concern.

How to cite this article: Gross JM, Nascimento GG, Araújo VC, Bönecker MJS, Furuse C. Mini-implants for Orthodontic Anchorage: Surface Analysis after Redrilling and Sterilization -An in vitro Study. J Contemp Dent Pract 2016;17(4):300-305.

Source of support: Nil

Conflict of interest: None

\section{INTRODUCTION}

Biomaterials are used for a variety of long-term purposes in the body, and the most widespread material used is titanium. Titanium is used in dental and orthopedic implants, mainly because of its excellent biocompatibility ${ }^{1}$ and corrosion resistance. ${ }^{2}$ Osseointegrated implants are considered reliable sources of anchorage for orthodontic treatment, ${ }^{3,4}$ however, their large size can restrict their use. To overcome this problem and to minimize the surgical trauma, mini-implants were developed. ${ }^{5,6}$ Besides the size, mini-implants present many advantages, such as minimal anatomic limitations, minor surgery, increased patient comfort, immediate loading, and lower costs. ${ }^{3,7}$

Mini-implants are usually used for acute/temporary reasons, such as bone anchorage and fracture fixation with plates, and after removal, the recovered devices are discarded. However, economic factors have caused some clinicians to reuse implants or other medical devices that are meant to be disposable. ${ }^{8,9}$ In dentistry, many devices are commonly reused, such as endodontic files or nickel-titanium (NiTi) rotatory instruments, even after penetrating in infected root canals, if an adequate protocol for sterilization is followed. ${ }^{10}$

Orthodontic treatment is still an expensive treatment not only due to long duration, probably years, but also because of the material involved. Whitesides et $\mathrm{al}^{11}$ verified that adults with high income visited more an 
orthodontist than adults with low income in the United States. A possibility of reducing costs from orthodontic treatment is to reuse some material, such as wires, ${ }^{12}$ molar bands, ${ }^{13}$ and mini-implants, in the clinical routine.

Reports on the reuse of medical devices after sterilization are scarce, and there is a concern about the consequences of systemic infection and mechanical properties of the involved material. While such devices may be used again in the same patient, ${ }^{14}$ residual endotoxin may be an obstacle for the clinical outcome. Certainly there are ethical considerations about devices reuse in different patients even taking into account the cost-benefit. ${ }^{8}$ Some studies suggest that certain implant materials can be used a number of times in humans. However, the results are largely anecdotal, so a critical assessment of the clinical response to reused implants is limited. Autoclave is a widely used method for sterilization in dental offices, and previous studies have assessed the effects of autoclaving on NiTi and beta-titanium arch wires, ${ }^{12}$ titanium implant cover screws, ${ }^{8} \mathrm{NiTi}$ alloy, ${ }_{1}^{15}$ discs of commercially pure titanium, ${ }^{16}$ and titanium miniplates and screws used in craniofacial reconstruction. ${ }^{17}$ Mattos et $\mathrm{al}^{18}$ observed in vitro that autoclave sterilization has no effects on miniimplants resistance to fracture.

As regards the retrieval of mini-implants, currently, there is little evidence on the profile of their surface during service, including structural alterations and changes in the mechanical properties. ${ }^{19}$ They can be decisive for miniscrew success, which relies upon stable fixation throughout usage, fracture resistance, as well as relative ease of removal. Immediate loading in the clinical setting could reduce osseointegration and lead to the soft tissues not enveloping the miniscrew surface. ${ }^{20}$ Previous reports, however, did not present data of surface alterations after multiples insertions in the bone as well as possible damages caused by the autoclave process.

Based on the above explanation, the main purpose of the present study was to investigate, in vitro, possible alterations on mini-implants surface after retrieval and if the cleaning process and sterilization can predispose damages.

\section{MATERIALS AND METHODS}

Two similar commercial brands of orthodontic conic self-drilling and self-dapping mini-implants ASTM F-136 grade $\mathrm{V}$ and Ti-6Al-4V were selected for this study. The samples $(\mathrm{n}=18)$ were divided into two groups:

1. Neodent Group (NG): Nine mini-implants Neodent Fix (Neodent, Curitiba, Brazil) with $9.0 \mathrm{~mm}$ length and $1.6 \mathrm{~mm}$ diameter (reference 109497; lot number 2839284)

2. Dentos Group (DG): Nine mini-implants AbsoAnchor (Dentos, Daegu, South Korea) with $8.0 \mathrm{~mm}$ length and
$1.6 \mathrm{~mm}$ diameter (reference SH1615-08; lot number MI71019C).

Briefly, to measure possible deformations of miniimplants' reuse, samples were drilled in artificial bone tissue, removed, and redrilled four times. Immediately, samples were analyzed by scanning electron microscopy (SEM), and surface alterations were verified through thread and pitches deformation.

To evaluate alterations caused by insertion/removal and also the influences of the cleaning process and sterilization, three mini-implants of each group (NG and DG) were submitted to three different procedures:

1. Complete sequence (insertions and sterilization), which simulates four redrilling of the mini-implant: Initial photomicrography (T_0); first insertion, removal and autoclave cycle; second insertion, removal, photomicrography (T_1), and autoclave cycle; third insertion, removal and autoclave; fourth insertion, removal and final photomicrography (T_2).

2. Sequence of insertions without sterilization: Three mini-implants from NG and three from DG were tested according to the following steps: Initial photomicrography (T_0); first insertion and removal; second insertion, removal and photomicrography (T_1); third insertion and removal; fourth insertion, removal and final photomicrography (T_2).

3. Sequence of sterilization without insertion: Initial photomicrography (T_0); first autoclave cycle; second autoclave cycle and photomicrography (T_1); third autoclave; fourth autoclave cycle and final photomicrography (T_2).

\section{Insertion and Removal of Mini-implants}

All 18 mini-implants were marked with a diamond bur KG2200 (KG Sorensen, São Paulo, Brazil) at the transmucosal region and at the head of the screw, to standardize the area to be photographed in SEM analysis. After that, implants were installed and removed in blocks of cortical and trabecular synthetic bone (Nacional Ossos, Jaú, Brazil) with dimensions $13 \mathrm{~cm} \times 18 \mathrm{~cm} \times 4 \mathrm{~cm}$, reproducing a type D2 bone according to the Misch classification. ${ }^{21}$

Insertion was made according to manufacturers' instructions with a Surgic XT motor (NSK, Zhengzhou, Japan) and Montblanc handpiece (Anthogyr, Sallanches, France) with reduction of $1 / 16$ in $300 \mathrm{rpm}$ and maximum preestablished torque of $15 \mathrm{~N} / \mathrm{cm}$ in a perpendicular angle. The handpiece was supported by a metallic arm to keep the its head always in the same angulation in relation to the bone block. Insertion was performed without previous perforation, only using the handpiece key, set on handpiece and motor (Fig. 1). Implant removal was performed in the same way, however, with reverse rotation. 


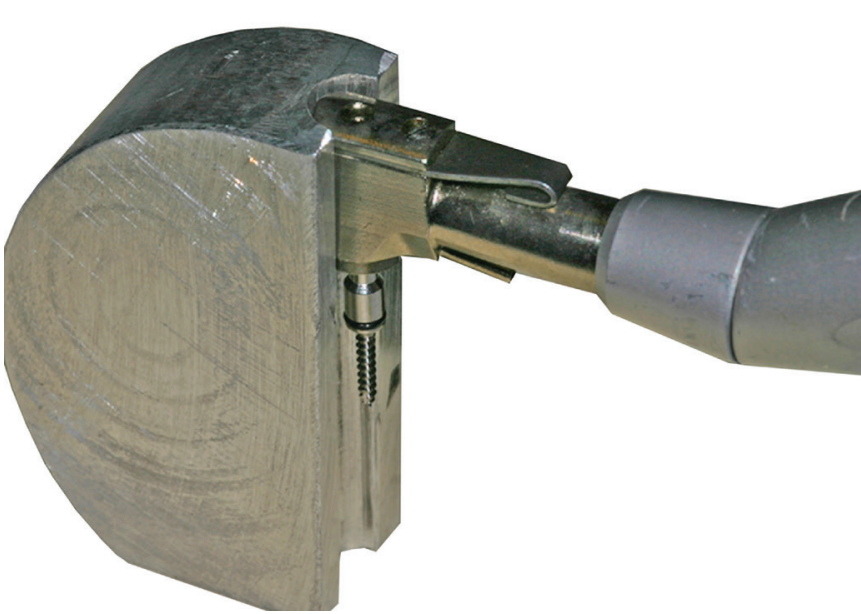

Fig. 1: Metallic device used to perforate the synthetic bone in order to maintain the same angulations

\section{Cleaning and Sterilization of Mini-implants}

After removal, mini-implants were cleaned by friction with a $70 \%$ ethanol solution, followed by ultrasonic cleaning in water (Cristófolli; Campo Mourão, Brazil) with enzymatic soap (10 minutes) with following sterilization in autoclave $170^{\circ} \mathrm{C}, 01 \mathrm{ATM}$ for 40 minutes (Dabi Atlante; Ribeirão Preto, Brazil).

\section{SEM}

A SEM (Superscan SSX-55, Shimadzu, Tokyo, Japan) was set at $20 \mathrm{kV}$ of acceleration and filament current at $0.8 \mu \mathrm{A}$. Mini-implants were mounted in an aluminum stub with a carbon double-sided adhesive with the bur mark facing up. Photomicrographs were then taken for posterior analysis.

\section{Analysis of Results}

A total of 220 photomicrographs were taken and visually analyzed in order to compare the surface characteristics between groups. Before the analysis, both examiners were submitted to a training with an expert. The training consisted in a practical demonstration of all the evaluated criteria. At the qualitative analysis, alteration of the miniimplant was verified in images under $50 \times$ magnification according to the following scores:

- No alterations;

- Mild: Presence of scratches and little deformation only on the top of threads;

- Moderate: Deformation all over the thread;

- Severe: Deformation all over the thread and alteration of the axis.

Quantitative analyses were performed under 100x magnification; it used the microscope software (Superscan SS-550, Shimadzu, Tokyo, Japan) in order to measure the distances between threads in three different regions across the active part of the mini-implants: (a) The first thread located immediately below the transmucosal profile;

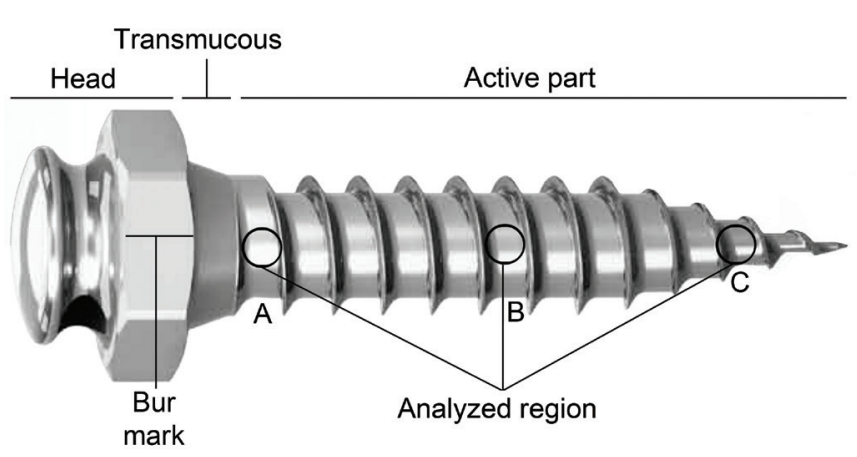

Figs 2A to C: Three regions analyzed of the mini-implants: (A) First; (B) intermediary; and (C) last region

(b) the second thread located in the intermediary region between the first and the last thread; (c) the last thread located in the active part (Figs 2A to C). All analyses were blindly conducted by two researchers independently (J.M.G. and G.G.N.). In order to verify the agreement intra- and interexaminers, a Spearman correlation index (r) was calculated for the quantitative measures; for the qualitative concordance, the kappa index was used.

Statistical analyses were carried out using Stata 11.0 (StataCorp, College Station, TX, USA). In the absence of a normal distribution, nonparametric tests were performed: Friedman for the paired comparison; Kruskal-Wallis for the intergroup comparison. A level of significance of $\mathrm{p} \leq 0.05$ was considered.

\section{RESULTS}

\section{Level of Agreement}

Spearman correlation $(r)$ for the quantitative analyses was 0.87 interexaminers and 0.81 intraexaminers. For the qualitative analyses, the kappa values were 0.81 and 0.83 respectively. All values were considered acceptable and demonstrated a high level of agreement between the examiners.

\section{Qualitative Analysis}

Qualitative analysis of NG demonstrated, initially before any insertion, a regular surface with a few imperfections at the apex of the active part in five implants (Figs 3A and $B$ ). After the second and fourth reinsertions, no visible deformations were observed compared to the initial photomicrographs. In relation to DG, no apparent deformation was noted initially. However, after second reinsertion, a small deformation on some threads was noted in all the nine mini-implants (Figs 3A and B). From second to forth reinsertions, no additional alterations were seen. During the drilling and removal, tensions were produced in the head of the mini-implants that may have brooked them. So, this region was also analyzed and no deformation was noted in both groups (Figs $3 \mathrm{~A}$ and B). Nevertheless, the presence of synthetic bone could be noted in some samples (Figs 4A to F). 

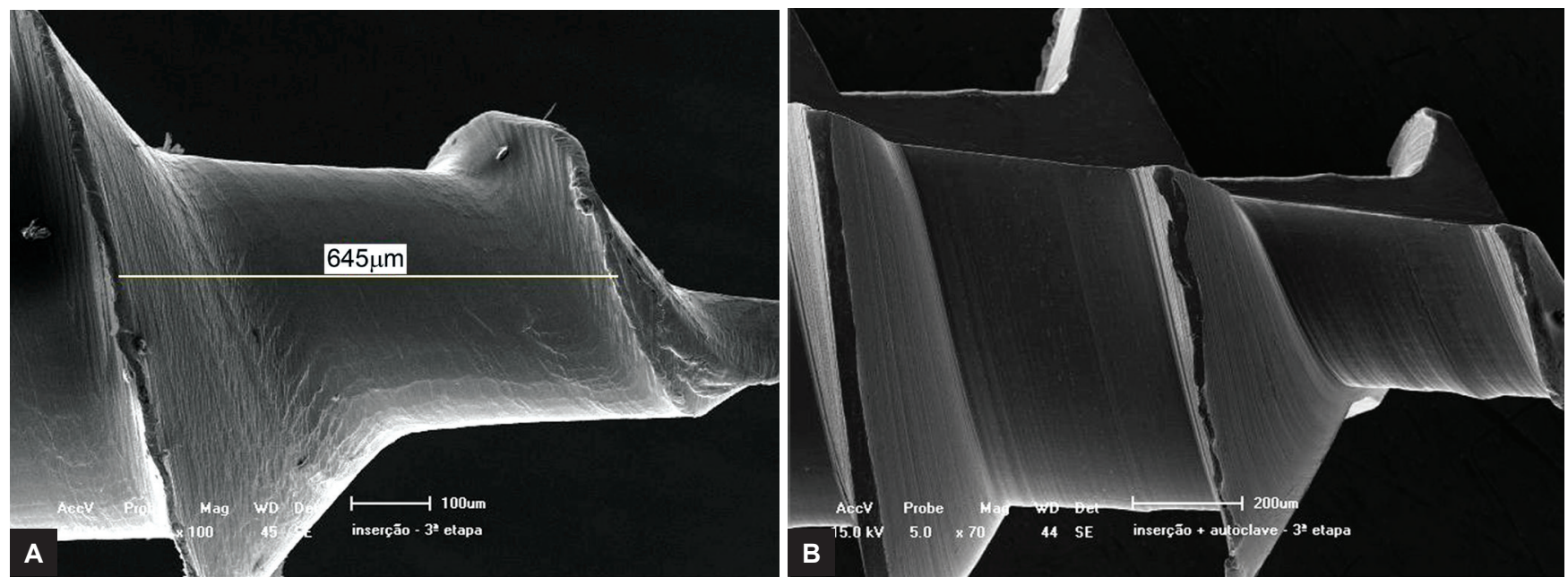

Figs $3 A$ and B: Scanning electron micrographs of mini-implant before any insertion in the synthetic bone: (A) Mini-implant of the Neodent Group (NG), (B) mini-implant of the Dentos Group (DG). Small deformations are noted in both groups

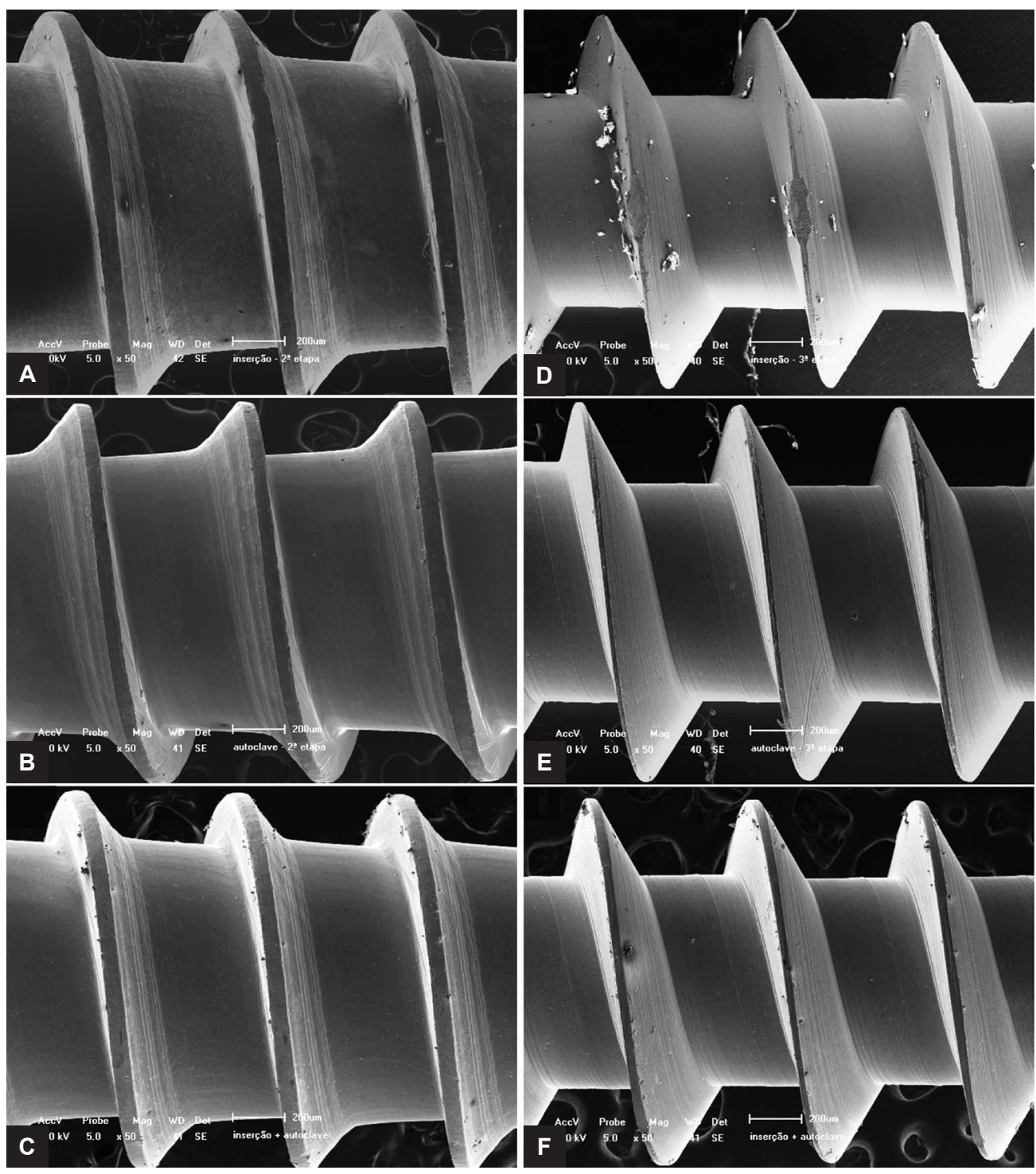

Figs 4A to F: Scanning electron micrograph: (A) Mini-implant from Neodent Group (NG) analyzed after the second insertion only; (B) Mini-implant from NG analyzed after the second sterilization only; (C) Mini-implant from NG analyzed after the second insertion combined with sterilization; (D) Miniimplant from DG analyzed after the second insertion only; (E) Mini-implant from GD analyzed after the second sterilization only; and (F) Mini-implant from GD analyzed after the second insertion combined with sterilization. Small pieces of artificial bone are noted in Figures $D$ and $E$ 
Table 1: Mean distances and standard deviation values between threads among groups according to procedures, mini-implant region, and sequence of exposition, Campinas, Brazil

\begin{tabular}{|c|c|c|c|c|c|c|c|c|c|c|}
\hline & \multicolumn{3}{|c|}{$T \_0$} & \multicolumn{3}{|c|}{$T_{-} 1$} & \multicolumn{3}{|c|}{$T \_2$} \\
\hline & & \multicolumn{3}{|c|}{ Intermediary } & \multicolumn{3}{|c|}{ Intermediary } & \multicolumn{3}{|c|}{ Intermediary } \\
\hline & & Initial region & region & Last region & Initial region & region & Last region & Initial region & region & Last region \\
\hline \multirow[t]{3}{*}{ DG } & $A$ & $672.3(35.8)$ & $620.3(15.5)$ & $613.6(8.1)$ & $691.7(2.5)$ & $663.7(28.9)$ & $642.7(17.2)$ & $700.3(11.9)$ & $676(17.1)$ & $660.3(18.2)$ \\
\hline & $B$ & $652(21.7)$ & $641.3(26.5)$ & $631.7(21.0)$ & $664(1.7)$ & $632(27.5)$ & $646.7(7.1)$ & $680.3(26.1)$ & $626.7(19.0)$ & $634(12.8)$ \\
\hline & C & $635.7(18.4)$ & $662(23.1)$ & $635.3(19.8)$ & $637.7(16.6)$ & $669.3(24.8)$ & $647.3(22.1)$ & $636.7(22.0)$ & $665.7(18.5)$ & 626.7 (15.9) \\
\hline \multirow[t]{3}{*}{ NG } & $A$ & $646.3(28.0)$ & $672.7(18.0)$ & $672.3(25.4)$ & $670(13.1)$ & $677.7(9.45)$ & $700(5.2)$ & $669.7(13.4)$ & $680(23.5)$ & $690.7(11.1)$ \\
\hline & B & $623.7(14.2)$ & $641(13.0)$ & $639(13.2)$ & $632.7(10.1)$ & $648.7(12.7)$ & $626(27.2)$ & $657(3.5)$ & $681(12.8)$ & $655.3(14.5)$ \\
\hline & C & $677.7(12.4)$ & 625 (23.3) & $634.7(25.9)$ & $696(27.2)$ & $642.3(13.3)$ & $667.7(26.4)$ & $691.7(13.0)$ & $642.317 .8)$ & $627.7(7.6)$ \\
\hline
\end{tabular}

DG: Dentos Group; NG: Neodent Group; A: Insertion and sterilization; B: Sterilization only; C: Insertion only; T_0: Before any procedure; T_1: After two cycles; T_3: After four cycles

Mann-Whitney, Kruskal-Wallis, and Wilcoxon tests: $p>0.05$

\section{Quantitative Analysis}

The mean distances between implant threads were similar after all experimental events (insertions and sterilization, only insertions, and only sterilizations), in the three regions of the mini-implants analyzed (initial, intermediary, and final portions) of both groups (NG and DG), in the initial (T_0), after second (T_1), and after forth (T_2) reinsertions photomicrographs. Implants of T_0 were considered the control. Statistical analysis did not show significance $(p>0.05)$ when these values of the NG were compared to the values of the DG, and also when the mini-implants of the same group, NG or DG, were compared (Table 1).

\section{DISCUSSION}

Our data demonstrated that reuse until four times and sterilization of mini-implants did not significantly alter the surface structure in vitro. Moreover, sanitizing process with friction, ultrasonic cleaning, and autoclave were not effective to remove all artificial bone tissue from the pitches of mini-implants.

Devices that can be cleaned and resterilized without modifying their structural integrity and function may be used more than once and are safe and cost-effective. ${ }^{14,22}$ The use of a steam autoclave has long been advised as a means of sterilization of instruments used in dental surgery. ${ }^{23}$ Nevertheless, repeated cycles of steam heat can damage dental instruments for corrosion ${ }^{24}$ or deformation by alteration of mechanical properties. ${ }^{25}$ Although our results did not show significant alterations on the surface of the mini-implants after four cycle of sterilization, Thierry et $a{ }^{15}$ showed that autoclaving could modify the surface topography and chemical surface of NiTi alloys. Mattos et $\mathrm{al}^{18}$ studied, in vitro, the effects of autoclave sterilization in fracture resistance of mini-implants and did not find differences on the mechanical properties.
Moreover, a previous in vivo study observed that, even with surface alterations, clinical outcomes of coverscrews were not changed. Adelson et $\mathrm{a}^{17}$ tested titanium plates and screws used in craniofacial reconstruction in 10 and 50 cycles of autoclaving and compared mechanical properties values of these groups with a control group without autoclaving. The authors found that although there was a trend toward decreasing the flexural strength and increasing the possibility of implant fracture as the number of autoclave cycles increased, this did not mean statistical significance. Therefore, they concluded that repeated cycles of autoclaving had no significant effect on the integrity of titanium plates and screws.

Despite of the biological and mechanical security of the sterilization process, our results indicate that dental mini-implant pitch was difficult to clean and synthetic bone tissue residues were observed on some samples. This is corroborated by the results of Sennerby et al, ${ }^{26}$ who performed the same cleaning protocol used in our study, showing that even carefully cleaning and sterilizing the devices was not effective to remove bone residues. Schwartz et al, ${ }^{8}$ however, demonstrated that an ultrasonic procedure involving soap and water for 10 minutes, butanol for 10 minutes, and $95 \%$ ethanol twice for 10 minutes, followed by steam autoclaving at $170^{\circ} \mathrm{C}$ for 40 minutes, was effective for cleaning coverscrews.

There are few studies in the literature about miniimplants surface after insertion. Choi et $\mathrm{al}^{27}$ evaluated the surface roughness of two different types of miniimplants and did not find any definite alteration, corroborating with our findings. However, Eliades et $\mathrm{al}^{19}$ observed surface alterations in retrieved devices, such as loss of gloss in some parts. It is important to highlight that even with these possible alterations, the tested mini-implants did not present significant differences in mechanical properties, which is in accordance with our findings. 
As the first study to evaluate multiple retrieves and sterilization with autoclave of mini-implants, this study presents as main weakness the in vitro design. Therefore, we cannot be absolutely sure that the results can be translated to the clinical context; however, the advantage of an in vitro experiment is that it allows controlling the number of external variables.

\section{CONCLUSION}

The results of this study suggest that the tested miniimplants can be retrieved without damage to their surface after four cycles of insertion, removal, and sterilization. It is important to highlight that usually miniimplants are retrieved in the same patient only changing the installation site; thus, an incomplete removal of bone tissue between threads does not represent a biological concern. However, more studies should be conducted with different protocols to improve bone removal. Also, in vivo tests should be carried out to evaluate tissue response to cleaned and sterilized implants.

\section{REFERENCES}

1. Xavier SP, Carvalho PS, Beloti MM, Rosa AL. Response of rat bone marrow cells to commercially pure titanium submitted to different surface treatments. J Dent 2003 Mar;31(3): 173-180.

2. Cheng Y, Hu J, Zhang C, Wang Z, Hao Y, Gao B. Corrosion behavior of novel Ti-24Nb-4Zr-7.9Sn alloy for dental implant applications in vitro. J Biomed Mater Res B Appl Biomater 2013 Feb;101(2):287-294.

3. Roberts WE, Helm FR, Marshall KJ, Gongloff RK. Rigid endosseous implants for orthodontic and orthopedic anchorage. Angle Orthod 1989 Winter;59(4):247-256.

4. Odman J, Lekholm U, Jemt T, Branemark PI, Thilander B. Osseointegrated titanium implants - a new approach in orthodontic treatment. Eur J Orthod 1988 May;10(2):98-105.

5. Kanomi R. Mini-implant for orthodontic anchorage. J Clin Orthod 1997 Nov;31(11):763-767.

6. Miyawaki S, Koyama I, Inoue M, Mishima K, Sugahara T, Takano-Yamamoto T. Factors associated with the stability of titanium screws placed in the posterior region for orthodontic anchorage. Am J Orthod Dentofacial Orthop 2003 Oct;124(4):373-378.

7. Fritz U, Ehmer A, Diedrich P. Clinical suitability of titanium microscrews for orthodontic anchorage-preliminary experiences. J Orofac Orthop 2004 Sep;65(5):410-418.

8. Schwartz Z, Lohmann CH, Blau G, Blanchard CR, Soskolne AW, Liu Y, Cochran DL, Dean DD, Boyan BD. Re-use of implant coverscrews changes their surface properties but not clinical outcome. Clin Oral Implants Res 2000 Jun;11(3):183-194.

9. Lavallee DJ, Lapierre NM, Henwood PK, Pivik JR, Best M, Springthorpe VS, Sattar SA. Catheter cleaning for re-use in intermittent catheterization: new light on an old problem. SCI Nurs 1995 Mar;12(1):10-12.
10. King JB, Roberts HW, Bergeron BE, Mayerchak MJ. The effect of autoclaving on torsional moment of two nickel-titanium endodontic files. Int Endod J 2012 Feb;45(2):156-161.

11. Whitesides J, Pajewski NM, Bradley TG, Iacopino AM, Okunseri C. Socio-demographics of adult orthodontic visits in the United States. Am J Orthod Dentofacial Orthop 2008 Apr;133(4):489.e9-489.e14.

12. Pernier C, Grosgogeat B, Ponsonnet L, Benay G, Lissac M. Influence of autoclave sterilization on the surface parameters and mechanical properties of six orthodontic wires. Eur J Orthod 2005 Feb;27(1):72-81.

13. Dowsing P, Benson PE. Molar band re-use and decontamination: a survey of specialists. J Orthod 2006 Mar;33(1):30-37.

14. Karch M, Alt E, Schmitt C, Schomig A. Reimplantation of an infected, abdominally implanted defibrillator in the subpectoral region. J Cardiovasc Surg (Torino) 1996 Feb;37(1):67-70.

15. Thierry B, Tabrizian M, Savadogo O, Yahia L. Effects of sterilization processes on NiTi alloy: surface characterization. J Biomed Mater Res 2000 Jan;49(1):88-98.

16. Vezeau PJ, Koorbusch GF, Draughn RA, Keller JC. Effects of multiple sterilization on surface characteristics and in vitro biologic responses to titanium. J Oral Maxillofac Surg 1996 Jun;54(6):738-746.

17. Adelson RT, DeFatta RJ, Ducic Y. Integrity of craniofacial plating systems after multiple sterilization procedures. J Oral Maxillofac Surg 2007 May;65(5):940-944.

18. Mattos CT, Ruellas AC, Sant'anna EF. Effect of autoclaving on the fracture torque of mini-implants used for orthodontic anchorage. J Orthod 2011 Mar;38(1):15-20.

19. Eliades T, Zinelis S, Papadopoulos MA, Eliades G. Characterization of retrieved orthodontic miniscrew implants. Am J Orthod Dentofacial Orthop 2009 Jan;135(1):10.e1-10.e7.

20. Sebbar M, Bourzgui F, Aazzab B, Elquars F. Anchorage miniscrews: a surface characterization study using optical microscopy. Int Orthod 2011 Sep;9(3):325-338.

21. Misch CE. Bone classification, training keys to implant success. Dent Today 1989 May;8(4):39-44.

22. Sonis AL. Air abrasion of failed bonded metal brackets: a study of shear bond strength and surface characteristics as determined by scanning electron microscopy. Am J Orthod Dentofacial Orthop 1996 Jul;110(1):96-98.

23. Palenik CJ, Burke FJ, Coulter WA, Cheung SW. Improving and monitoring autoclave performance in dental practice. $\mathrm{Br}$ Dent J 1999 Dec 11;187(11):581-584.

24. Benyahia H, Merzouk N, Ebn Touhami M, Zaoui F. Effects of sterilization and disinfection procedures on the corrosion of orthodontic ligature cutters. Int Orthod 2012 Mar;10(1):1-15.

25. Canalda-Sahli C, Brau-Aguade E, Sentis-Vilalta J. The effect of sterilization on bending and torsional properties of K-files manufactured with different metallic alloys. Int Endod J 1998 Jan;31(1):48-52.

26. Sennerby L, Lekholm U, Ericson LE. Soft-tissue response to clinically retrieved titanium cover screws reimplanted in the rat abdominal wall. Int J Oral Maxillofac Implants 1989 Fall;4(3):233-239.

27. Choi SH, Cha JY, Joo UH, Hwang CJ. Surface changes of anodic oxidized orthodontic titanium miniscrew. Angle Orthod 2012 May;82(3):522-528. 